\title{
Earth's Magnetic Field and Cosmic Radiation in CNS Function: Anomalous Magnetic Fields, Cause of Mental Diseases
}

\author{
Trifunovic Nikola1 ${ }^{1}$, Vladislav Cizmic ${ }^{2}$, Racic Alek ${ }^{3}$ \\ ${ }^{1}$ Retired Geophysicist, Belgrade, Serbia \\ ${ }^{2}$ Faculty of Dentistry, Pancevoe, Serbia \\ ${ }^{3}$ Klinicki Centar Srbije, Belgrade, Serbia \\ Email: ntrifunovic41@yahoo.com
}

Received 2 November 2014; revised 18 December 2014; accepted 7 January 2015

Copyright (C) 2015 by authors and OALib.

This work is licensed under the Creative Commons Attribution International License (CC BY). http://creativecommons.org/licenses/by/4.0/

(c) $\underset{\mathrm{EY}}{\mathrm{B}}$ Open Access

\section{Abstract}

Biosphere is characterized by the existence of Earth's magnetic field (EMF) and cosmic radiation (CR). By measuring the EMF in beds of mentally ill people (MI), anomalous magnetic fields (AMF) were measured. That's why we approached the study of central nervous system (CNS). Aims: To explain physiology of the CNS, how to use cosmic radiation (CR) in occurring brain micro power; To prove that neurons have magnetic characteristics; To explain brain amplifier and capacitor; To explain brain waves, alertness and sleep-REM phase, memory and reproduction as well as congenital instincts; To clarify consciousness; To determine correlation between AMF and MI people's heads; If possible, to keep track of the health status of patients after relocation from AMF and to prove that the cause of MI people are AMF. Methodology: Literature study of the physiology and functioning of the CNS. By knowing physics, electric engineering and biochemistry, we explained the functioning of the CNS. Measuring of AMF was performed with proton magnetometer in 19 beds of MI people (depression, schizophrenia, epilepsy and Parkinson's disease) and in 2 beds of healthy people. Geophysical interpretation of measured values was performed and the results are shown on sketches. MI people were transferred in natural EMF and their health status was monitored. Results: Physiology of the CNS and goals were explained. The brain generates micro electric power using CR. The cerebrum is the capacitor. The brain is the amplifier of afferent and efferent micro power impulses. The electroencephalogram, the emergence of brain micro powers, brain waves, alertness and sleep-REM phase, memory and reproduction as well as congenital instincts were explained. The consciousness was defined. AMF balance in MI people's beds is presented on sketches. By measuring, spatial correlation between AMF and the patient's head is determined, which indicates the cause of MI people. Monitoring of the health status of patients after relocation from AMF, did not give reliable recovery results due to the short monitoring time of patients, except in epilepsy. Conclusion: The CNS with CR is generator of micro electric power in the body. The main function of the CNS is explained. The correlation between AMF and MI people's heads on 


\begin{abstract}
about 500 patients was determined. With the help of psychiatrist, it's necessary to determine how much of a help, in treatment of MI people, living in natural EMF is. The causes of MI people are AMF in people's living area.
\end{abstract}

\title{
Keywords
}

Magnetized Neurons, AMF, Brain Capacitor, Brain Amplifier

\author{
Subject Areas: Neurology
}

\section{Introduction}

The characteristics of Earth, as a planet, is the existence of Earth magnetic field (EMF) and CR. EMF is a medium in which the bioworld lives and it magnetises everything that can be magnetised in biosphere. Production and moving of electricity happens due to the existence of CR and EMF. Electric fields occur thanks to CR which, by ionisation, produces electrons and ions in magnetosphere, i.e. in biosphere. A part of unabsorbed CR comes to the very Earth's surface and into the Earth crust.

The CR's quantity of electrons production mainly depends upon oxygen concentration.

"EMF and CR are the most important factors in the external environment interaction—bioworld's evolution" [1].

Molecules in the bioworld are characterised by susceptibility and electric conductivity and EMF gives them magnetisation.

The natural electric field source and potential differences in a cell and its surrounding is of periodical character. Fields can be considered as quasi stationary, while electricity is direct. Magnetic fields and temperature have a substantial effect upon movements of electrified particles. "In this way potential differences occur from around $50 \mathrm{mV}$ to around $70 \mathrm{mV}$ in front of and behind a cell's membrane” [2]. Negativity inside cells (in cytoplasm) is mostly generated by $\mathrm{P}$ (potassium-40), because it is radioactive, and gamma quants produce electrons.

Substances out of cell are transported by magnetic force lines (drift) in the cell. Magnetic field of organelles causes convectional movement of content. Separately, molecules enter cytoplasm and, selectively, into organelles. There happen biochemical reactions, temperature, electric voltage and magnetic properties change. Because of the magnetic properties change, movements directions change, too, so we get convectional movement of the substances of the opposite direction. For example, K-Na-ATP pump [1].

\section{The Central Nervous System (CNS)}

Our senses register all our experiences, then the relays of reticulo thalamic tract and, finally, the multi layered cerebral cortex.

Cosmic radiation (CR) produces electrons and ions which move through neuron nets. Brain contains ten times more oxygen than other organs, making its potential high. Therefore, the current flows all the time towards autonomous organs and peripheral nerves (lower potential). In this way brain gets rid of the redundant potential and body has electricity.

Since micro electricity is made wherever there are neurons because they are enriched with oxygen (4 times more than the white matter of the brain), CR makes clouds of electrons and ions which move by axons, all the regions being connected with nervous fibres. In this way we get movements directions of micro electricity, in all directions, what is manifested in the form of inhibition effect of one neuron upon other and the opposite-micro electricity of the same direction produces excitation. This is the clear explanation of the different behaviour of neurotransmitters, since the same ones behave both as inhibitors in one part of brain and as excitators in another brain region. Therefore, all this is enabled by micro electricity, as well as by excretion of neuro transmitters. This excretion of various neurotransmitters comes from different regions in CNS, i.e. cells, with axons and synapses being with different electric, magnetic, biochemical etc. Properties, that's why they secrete various substances. 


\subsection{Magnetic Properties of Neurons}

"Magnetisation centers are in the nucleus of all cells and the strongest are in the neurons, because they have a very thin cytoplasm, most oftenly none, but only the cell’s nucleus” [1].

Proofs that nervous cells magnetic forces are the most important characteristics for CNS functioning are following:

1) Low EMF affect the astronauts in cosmos brains in that way that they cannot remember their past experiences and have to write them down. The reasons are low quantities of EMF on higher altitudes.

2) Effects of high EMF upon CNS are, also, the appearance of "polar lunacy" with people who spend long time in the Earth poles.

3) Blood insufficiency in brain causes some regions to stop functioning normally (after brain stroke). Reducing the brain's temperature of diseased region causes strenghtening of inter molecular magnetic properties in neurons and there are no functional brain damages.

4) CNS neurons use glucose $(G)$ as energy without insuline [3], what clearly points that magnetic properties are stronger in neurons comparing to all other cells of an organism which needs insulin as a carrier, because together (glucose and insulin) get stronger magnetic properties and in that way they enter the cells [4].

$\mathrm{G}$ is a significant paramagnetic. The proof for it is that in blood $6 \%$ of glicose connects hemoglobin-Fe, in fact, magnetite. With the increase of $\mathrm{G}$ in blood this percentage increases proportionally [3]. The conclusion is that neurons have different magnetic properties.

\subsection{Brain Amplifiers (BA)}

"Neurons in pyramidal tract have been found as gigantic pyramidal cells. There are, as well, giganto cellular neurons in reticular excitational region” [3]. These gigantic neurons are amplifiers of the somatosensors and brain micro electric coded currents in the pyramidal motor cortex and in the reticular excitatory pons and mesencephalon region. As their dimensions are big, they have much stronger magnetization that is why they work as BA for the nervous impulses.

\subsection{Brain Condenser (BC)}

It is known that we know about cerebral cortex functioning the least, although it is the largest part of the nervous system. "Lamina cortex-a multi layered and columnar vertical cortex structure have six layers of neocortex... and ten on fifth to ten on sixth colonar substructures" [5]. This kind of layered construction of various dielectric constants, enables the cerebral cortex to function as BC which fills and empties the organism representing a rules of CNS functioning. The quantity of electrification in BC changes depending on the brain state, for example, in the awaken state it decreases because micro electric currents decrease and in the slow wave sleep charge quantity in BC increases.

The slow wave sleep occurs because in awaken state BC has got empty, so then occurs deep sleep and all body functions minimize, while CR generates electrons and ions all the time filling BC. When BC if full, brain goes into the REM phase of sleep characterised by fast eyes movements, dreaming and activity of muscles, because the excess potential appears in BC lasting from 5 - 30 minutes and returning every 90 minutes. CR has regenerated the excess of electric potential in BC, so the electricity is spent for the organism's activities in the REM sleeping phase.

"Cycles of sleeping-being awake have not been explained so far" [3]. We hope that the explanation about $\mathrm{BC}$ is clear.

\subsection{Brainwaves}

“The question of electroencephalogram (EEG) origin is still opened” [5]. In fact, a clear proof of everything presented is EEG. Electric registration of EEG shows a continual electric activity in brain.

"Brainwaves intensity on the scul's surface range between 0 - 200 micro Volts per second and their frequences over $50 \mathrm{~Hz}$ " [3] rain waves are mostly irregular. Clear approximate appearance of brainwaves is a shower of CR, which are also of various intensities and frequencies, being one oscillation in many seconds up to $50 \mathrm{~Hz}$, i.e. irregular.

1) Alpha (Figure 1) waves are with frequencies 8 - $13 \mathrm{~Hz}$, and their voltage is usually around 50 micro Volts, 
than brain is in awaken state and inactive.

2) Beta (Figure 1) waves are with frequences 14 - $80 \mathrm{~Hz}$. This brain activity increases frequences of breathing, heart activity and impulsive supply of brain with oxygen. In the extensive CNS activity (awaken state) spending electric energy is increased, so the fall of voltage decreases.

3) Delta (Figure 1) waves with frequency below $3.5 \mathrm{~Hz}$ occur in a very deep sleep, so the BC on this occasion fills up because electricity consumption is minimized. Breathing frequency, heart activity and impulsive oxygen supply decrease, and CR increases voltage to 200 microV.

Detection of alpha waves, when eyes are open (Figure 2), shows that occipital lobe drastically decreases voltage (uses more electric energy) and increases frequency (increased impulsive supply of oxygen to CNS), while closed eyes save electric energy, so waves with higher voltage and lower $f$ appear. Having said this, we have fully explained EEG.

Transection of nerves fibres from thalamus to cortex does not cause blocking of delta waves in cortex, therefore, delta waves continue to be produced in cortical neurons, what points that the dominant generators of micro electric potentials are in the six layered brain cortex, which functions as the brain capacitor.

\subsection{Psychic Functions}

"CNS consists of complex electric and magnetic activities. All these activities in neurons have bioelectrical and biochemical basis, being perception, thinking, emotions, learning and remembering” [6].

"Thinking, consciousness, memory, remembering and recall are still unexplained" [3].

1) Memory (learning and remembering) is a dynamic property of brain as whole, it is everywhere in brain, in fact, it is a coded magnetisation of neurons.

2) Learning by senses puts into movement a wave of electrons and ions, causing the synthesis of proteins in brain. There are two phases. Memory, which is created within a short time after learning occurs by electrons. "Electric currents, originated from the senses, stimulate neurons in brain to magnetise weakly and it quickly disappears" [6].

"For long lasting memories creation another wave is necessary after repetitions. In this phase, the dominant carriers of movements are ions which stimulate protein synthesis, and these are molecules in the form of magnetic dipoles which hook one to another and change the organisation of synapses in brain, representing a long lasting memory created by electronic and ionic currents in the cerebrum cortex" [6]. External effects-sight, hearing get into the cortex by microelectric currents created by electromagnetic fields which magnetise neurons.

Because of the additive magnetisation property, by repeating impulses of sensory currents, the magnetisation intensity is increased by magnetic induction in neurons and synapses, representing long lasting memory. As ex-

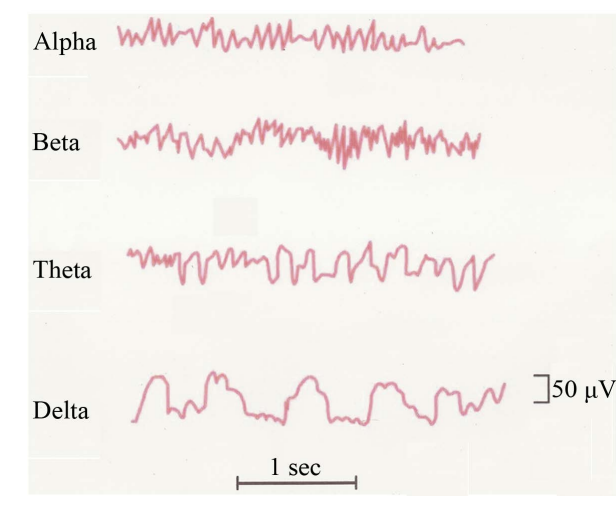

Figure 1. Brainwaves (EEG).

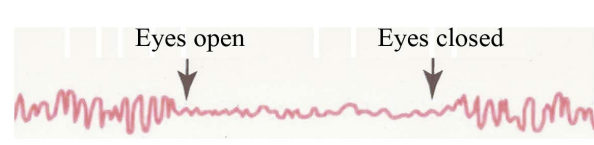

Brainwaves: Arthur C. Guyton, MEDICAL PHYSIOLOGY, 2011

Figure 2. Brainwaves of open and closed eyes. 
ternal sensory input via periphery receptors give coded (natural) electric impulses, it is normal that neurons magnetisation is also coded.

3) Recall—by external action on peripheral receptors or by association, a coded electric impulse is produced again what, resonantly, in neurons increases the coded magnetic induction (magnetic flux is changed) what creates micro currents in brain which are going into the senses (sight, speaking and motoric), and that is recall.

4) Spontaneous activity. Spontaneous brain activity characterises a high neuronal magnetisation, because reactions are automatic. "Currents from brain produced by potential of 10 - 100 micro Volts, with frequency ranging from $0-50 \mathrm{~Hz}$ and intensities of several micro Ampers, what depends on the brain conditions" [5]. Therefore, after long time of learning and exercising we have created the recognisable individual "styles" and behaviour.

With the wrong learning there can occur wrong understanding and attitude manifested in the form of paranoid behaviour of people.

5) The induced activity of brain is aroused by newly received information, "what induces potentials in cortex neurons from 2 - 3 microV” [5], producing very weak currents which, also, give a weak induced magnetisation in neurons nets, representing weakly memorised information.

All this is manifested in the form of our consciousness which we define as follows:

6) Consciousness is a micro coding electromagnetic wave created by electronic and ionic ultra low frequency (ULF) brainwaves, nets and aroused by methods of association, then by external affections from senses and internal interneural magnetic fields created by induction in the process of memorising and inherited instincts in the brain neuron nets [6]. Thoughts created in this way for reproduction are BA. Electromagnetic component being created by brain ULF electronic and ionic currents from brain neuron nets is strengthened.

7) Inherited functions. In CNS there are centers for all functions as well as inherited ones, like for example, motherhood, hunger, thirst, sex etc. Neurons of these centers are codedly magnetised by VMF during their creation (forming) yet in uterus. Specific senses (sensors for stimulation) after birth produce micro electric impulses which in neurons of certain centers create, through resonant electric fields, returning micro currents which are the response of CNS to external stimuli (what is completely the same as with the memorised information which automatically return to our senses). This micro currents stimulate motor functions, which satisfy the inherited necessities of an organism.

Even before a child's birth we can develop love for a certain kind of work which the person will perform in his or her life, what means that the person can be formed with inherited talent for various fields of work.

\section{Enormous Variable Magnetic-Magnetic (VM-M) Field, Anomalous Zones (Az) as Mental Diseases Cause}

Resulting mental disturbances and diseases are with high intensity of Az, which are product of our disregard with organization of our life in living spaces. By measuring VM-M field, it's been determined that everybody with mental illness, around 500, were spending day and night rest in anomalous VM-M fields, i.e. Az, Noting that no mentally ill people, that were living in natural EMF, were found.

\section{Measuring Results (Figure 3)}

The resulting intensity of total magnetic field vector was measured, while the variable magnetic field, as the perturbed field of EMF registered as: little present, present and highly expressed i.e. relative values.

In the attached sketches, on the beds of mentally ill people states of Az are shown. On sketches, Az with a rather variable magnetic field are isolated with adequate hatching.

There are four examples of measuring VM-M field, with four patients with epilepsy. (sc. 16, 17, 18 and 19 diagnosis given by appropriate health institutions in Belgrade) and two patients with Parkinson's disease (sc. 4 and 9). Increased VM-M field (unnatural) was registered within all patients. Patients with epilepsy were removed from Az to natural EMF, and that field is shown on sc. 7 and 13. After deployment, epilepsy didn't show up for many years. Sketches of mentally ill people (19 patients) and 2 healthy patients (sc 7 and 13). With all patients correlation between patient's head and $\mathrm{Az}$ is confirmed.

Presented examples in this paper are showing that this pathological state is caused by Az with a distinctive VM field. Disease occurs after a long stay in Az. Removing patient from Az can be helpful to the patient and the doctor in treatment. It must be noted that it is necessary to conduct clinical research in psychiatric clinics, where 
T. Nikola et al.

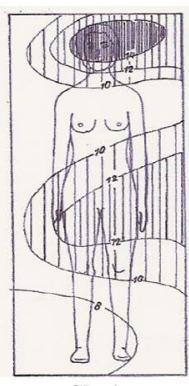

Sk. 1

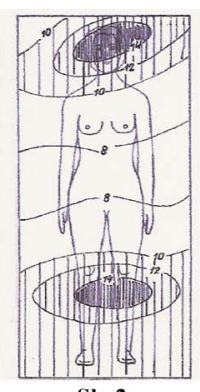

Sk. 2

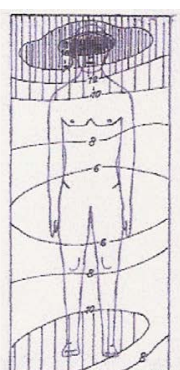

Sk. 3
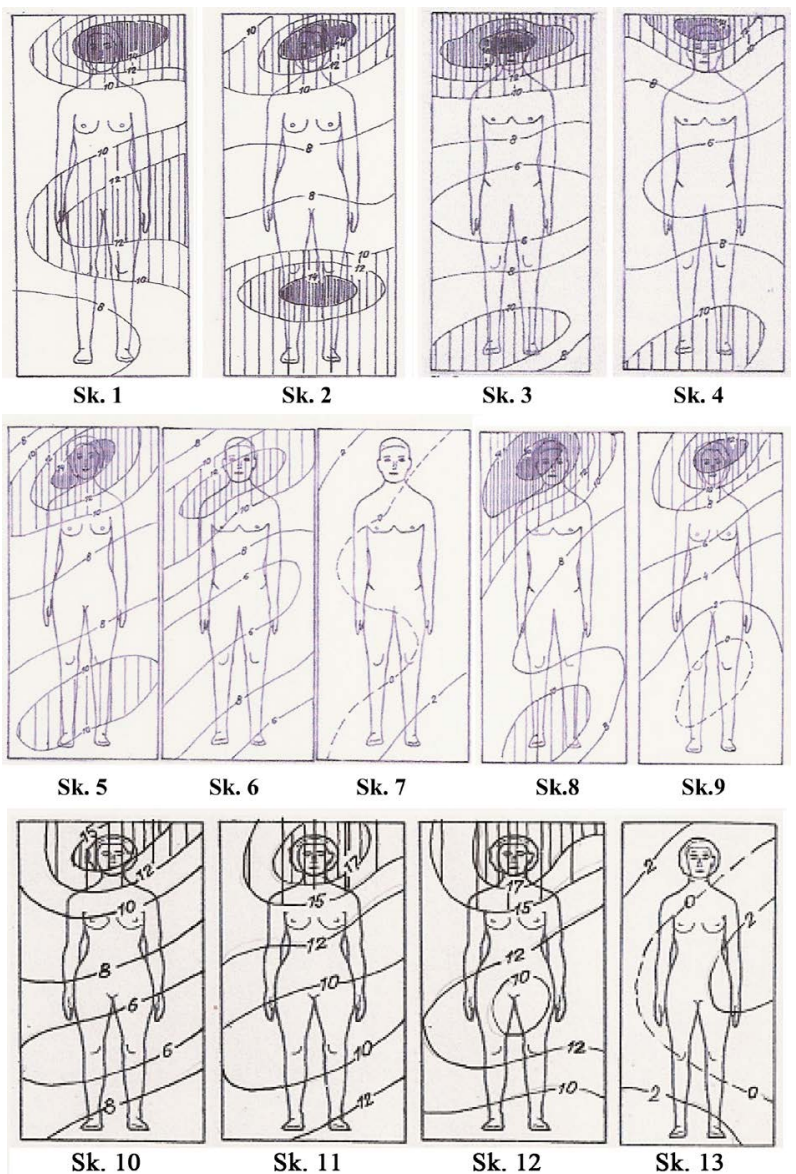

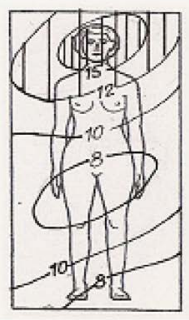

Sk. 14

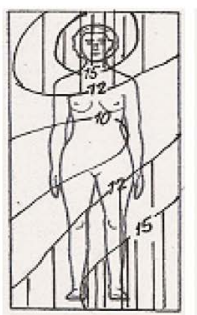

Sk. 18

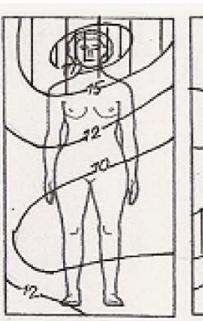

Sk. 15

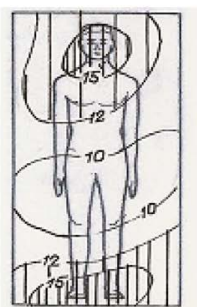

Sk. 19

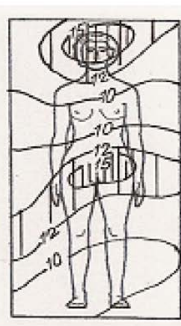

Sk. 16

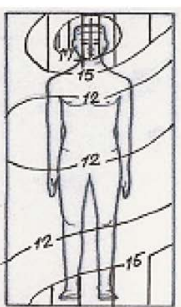

Sk. 20

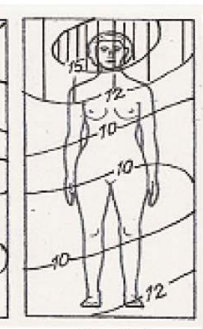

Sk. 17

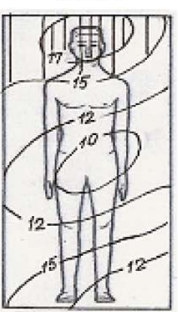

Sk. 21

L E G E N D

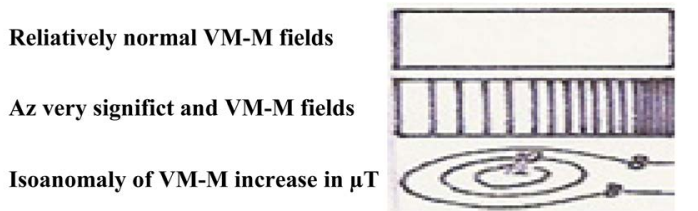

Figure 3. Sketches of measurement results. 
relevant professional teams must be included, to conclude how much of a help living in natural EMF with patient's treatment is.

Note: living in natural EMF, can be only useful, and in any way can't harm the patient.

VM-M field measurements in people's living spaces are performed more than 23 years. Based on the observation and monitoring the following is noticed: in homes where very distinctive Az are present, comes to bad influences on the CNS, and conflict and disagreements among family members are more often. Therefore, these environments leads to frequent divorces. It was also noticed that pupils and students who spend time in a relatively healthy environments i.e. spaces with natural values of EMF are very conscientious and working and less nervous in relation to those people who spend time in Az. It was also noted that, probably, there are optimal intensities for intellectual work which is about 44,000 nanotesla of this statement, there is a large number of examples of such proof.

\section{The Movement of the Most Developed Civilizations of People on Planet Earth}

A good example is the movement of the most developed civilizations of people on planet Earth. Which were created thanks to the changing values of EMF whose intensities enabled optimal development of the CNS.

It is necessary to give a theoretical explanation of the development of the most advanced civilizations that natural EMF's on Earth enabled. The civilizational development of human society dating back thousands of years BC. The first most advanced civilization was discovered around $6000 \mathrm{BC}$ in the valley of the Indus (Pakistan and India). Then, the center of planetary civilization moves towards today's Iran (Persian Empire) and Iraq. Around $3000 \mathrm{BC}$, Egyptian civilization emerges as the most developed in this period. Mentioned civilization were shifted from east to west of Asian-African continent. Further movement of the greatest scientific and cultural progress goes to the north. About 2000 years BC there has been a Greek civilization (Athens and Sparta). Around 1000 years BC, which covers the beginning of a new era, we have the Roman Empire. In VI, VII and VIII century we have the emergence of the Islamic religion with the most developed cities of the Mediterranean. In the fifteenth century, Spain and Portugal begin to develop. XIX, XX and the beginning of the XXI century is characterized by Western European, North American and Russian scientific and cultural civilization.

The movement of civilization was in the function of the human central nervous system (brain). In these intervals optimal intensity-flux of EMF for brain development was on the aforementioned areas. We are aware that changes of EMF dictate changes in living world, from the presented paper on the CNS, we can easily conclude that this movement of civilization was allowed by optimal flux of EMF on planet Earth.

It is known that in this time EMF is around the Equator and slightly wider than $20-30,000 \mathrm{nT}$, and the northern and southern latitudes are around $40-50,000 \mathrm{nT}$, at the poles it is about 60,000 and 70,000 nT. Middle latitude is $40-55,000 \mathrm{nT}$, and it's today's optimal intensity for the intellectual development of the people. This is a theoretical explanation of human development throughout the history of planet Earth. It is necessary to give an interpretation based on trends and developments of EMF and CR, what awaits us in the future.

It's known that promoter of cell division is EMF [1], it is also known that the same field weakens relatively quickly i.e. flux is smaller and smaller, and that according to some calculations, based on satellite tracking, in about 1300 years will reach zero. It is not difficult to assume what people can expect on Earth. The explanation is as follows: first, the reproduction of living world will disappear at latitudes around the equator, and then on the north and the south and at the end, life will also turn off near the poles. All of this presents the scientific explanation of the past and the future of living world on Earth.

\section{Discussion}

The authors of the work of the CNS are well aware that clinical research are the most important in medicine but to get to them, probably because the first author is geophysicist, was very difficult. It should be noted that currently there are small number of patients in Psychiatric hospital, thanks to psychiatrist who listened to a lecture of the first author at Serbian Medical Society in Belgrade. It can be safely concluded that the initial results are very good. As the research work with the mentally ill people is specific and it's difficult to implement, because it is essential that patients residing in the Earth's natural magnetic field, please note that studies of cancer and SVD was easier because of the placement of patients beds in natural EMF, where they usually spent day and night rest, and monitoring recovery was possible.

While the mentally ill people are very mobile and because of misunderstanding and unawareness they are 
looking for a typical AMF to enter into them with their head, because they are probably accustomed to spend day and night rest in such fields. Therefore, the main objective of these studies of measuring "in situ" was to determine the cause of the mentally ill people, so the large numbers of measuring of AMF was performed. After measuring of AMF in living area of more than 500 mentally ill patients, it is confirmed that there are no disease without typical AMF, where patient's head stays during day and night rest. Patients shown in this paper are of both sexes, treated in psychiatric clinics with discharged lists with diagnoses. Many of them didn't want to give the discharge lists. There for there were no interviews, just information from patients and their household that patient had mental difficulties. Four patients with epilepsy, two patients with Alzheimer disease and one with Parkinson's disease gave their discharge lists. It should be noted that there was no pressure on patients to give discharge lists or interview. Measuring in living area of mentally ill people was performed on patients or patient's relative's requests.

There is a small number of sketches, because they are mostly the same, the head is in typical AMF, and the disease is manifested after a long stay. Studying physiology and anatomy of the CNS, it was very clear that AMF with its variations from the environment, affects the micro powers that occur in brain and spread all over the body. That's why the explanation of main laws of the CNS functioning was written, and refers to the micropower generator, brain capacitor and amplifier and everything else shown in the work. When presented facts are accepted, than it should be admitted that paper is great innovation in biological science. It is clear that further research that are immensely promising, should proceed. In short, by regulating people to spend time mostly in the natural EMF would lead to the elimination of the most serious diseases of the twentieth century. Also, by regulating the magnetic field, which is very important, it's possible to educate and guide the young generation, and so on.

It is necessary to point out regarding the literature, that there are no such papers, as far as authors know, with similar explanations relating to the impact of physical fields on living world. Therefore, most authors refer to their published works on many World, European and Republican conferences and congresses, noting that scientific committees, as it is known, selected papers and invited the authors to present them. It is worth to say that the authors of these works did not pay the fee, to be able to display their works.

\section{Conclusions}

The brain is a generator of micro-currents throughout the body.

The CNS function is explained. All literature unexplained CNS functions are fully explained.

The causes of mental disorders are anomalous magnetic fields in people's living spaces.

The correlation between anomalous magnetic field-Az and head of the mentally ill people is determined. Living in the Earth's natural magnetic field is helpful to the patients and physicians in the treatment. It is necessary to carry out additional clinical research to determine the contribution of the living in the natural EMF to treatment of the mentally ill people: The etiological facts from the literature are not in contradiction with the results.

\section{References}

[1] Trifunovic, N. and Cizmic, V. (2014) Breathing Enable the Magnetic Properties of Erythrocytes (HEM Fe) Oxygen, Cells and Carbondioxide. Journal of Health Science, 2. www.davidpublishing.com/show.html

[2] Škokljev, A. (1998) Physiological Mechanisms of Acupuncture: The Psycho-Somatic Neuro Endocrine-Immune Aspects. Exogenous and Endoghene Biological Interactions, Center for Molecular Machines and IHIS FST Center, Belgrade.

[3] Guyton, A. (2003) Medical Physiology. Belgrade.

[4] Trifunovic, N. (2013) The Anomalous Increase of Magnetic Field-Cause of Atherosclerosis. The 6th European Congress for Integrative Medicine (ECIM), Berlin.

[5] Rakovic, D. (1995) Fundamentals of Biophysics. Belgrad.

[6] Trifunovic, N. and Komatina, S. (2003) Role of Enormous Changeable Earth Magnetic Fields (CEMF) Intensities in Etiopathogenesis of Mental Disturbances and Illnesses (MDI). Geophysics of the 21 Century-The Leap into the Future, Moscow, 1-4 September 2003. 